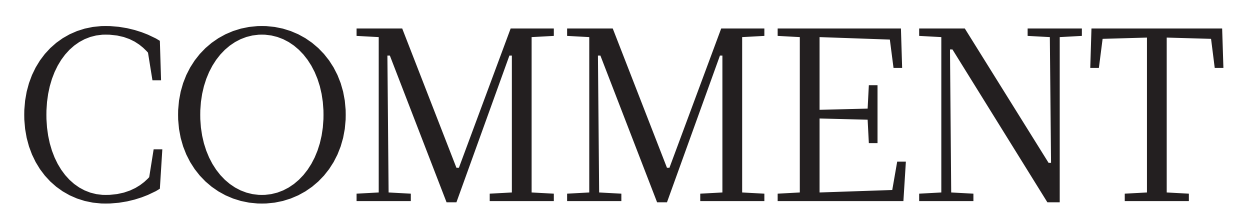

\begin{tabular}{ll|l|l}
\hline $\begin{array}{l}\text { ALTMETRICS Funding agency } \\
\text { rewards outputs other than } \\
\text { papers at last p.159 }\end{array}$ & $\begin{array}{l}\text { FICTION How science flows } \\
\text { through Herman Melville's } \\
\text { Moby-Dick p.160 }\end{array}$ & $\begin{array}{l}\text { puts inventors' personalities } \\
\text { centre stage p.162 }\end{array}$ & $\begin{array}{l}\text { Still worried about deferred } \\
\text { spending cuts p.163 }\end{array}$
\end{tabular}

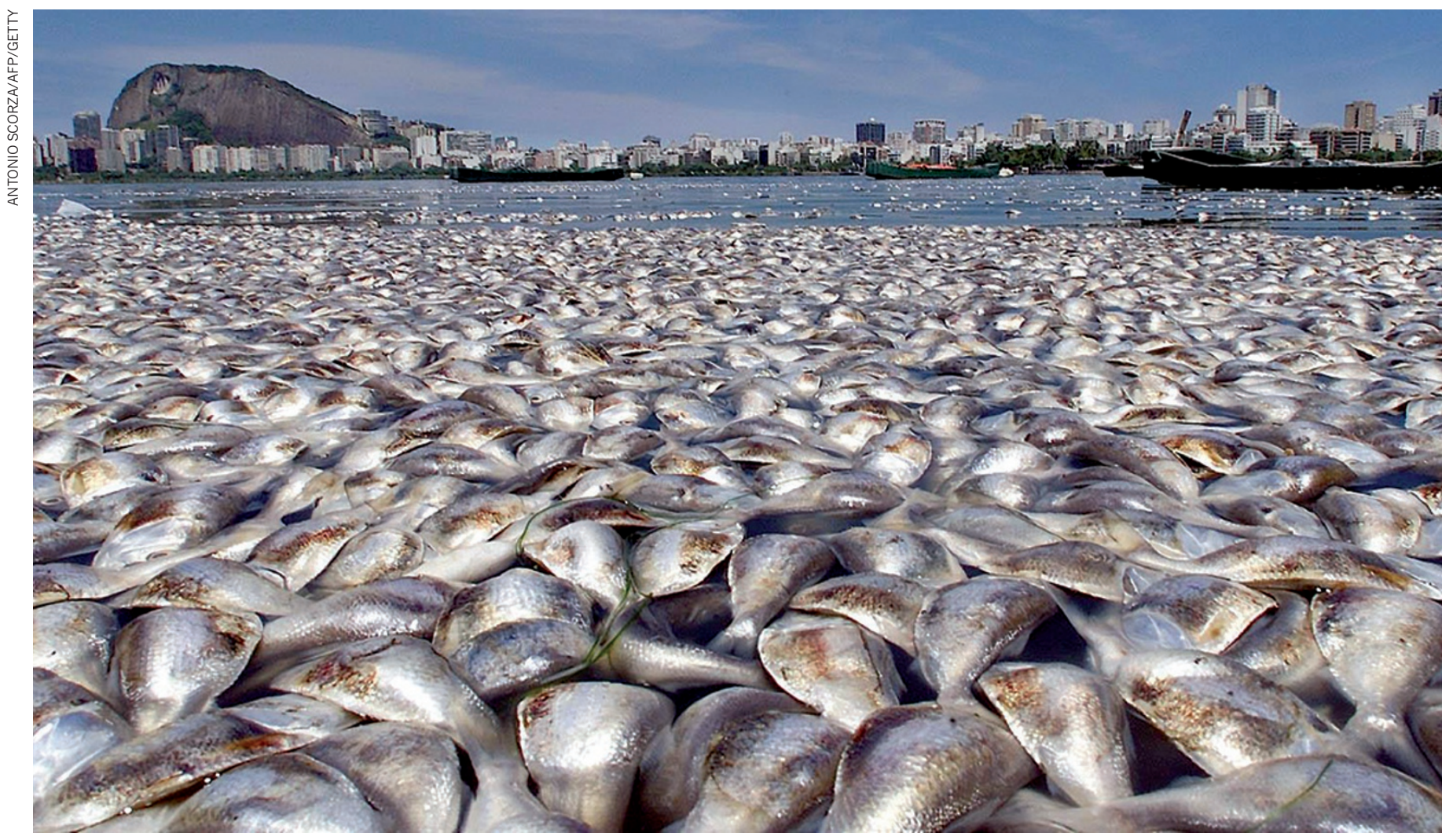

The shores of Rodrigo de Freitas Lake in Brazil are littered with fish killed by algal overgrowth in March 2000.

\title{
From patterns to predictions
}

Truly generic signals warning of tipping points are unlikely to exist, warn Carl Boettiger and Alan Hastings, so researchers should study transitions specific to real systems.

\section{$\mathrm{T}$} There has been much talk about tipping points over the past few years, and about the warning signals that may precede them. You could be forgiven for thinking that the forecasting of epidemics and stock-market crashes is just around the corner. But no one has yet managed to use the theory on early warning signals to predict a natural catastrophe.

The rewards of bridging the gap between the real world and mathematical conceptualizations of catastrophic shifts would be vast. Climate scientists might be able to foresee major shifts in the ocean currents with a rise in global temperatures; ecologists could potentially stave off pest outbreaks; and policies might be implemented to avert the collapse of fisheries ${ }^{1}$. (A report out this week from the World Economic Forum outlines other risks facing the world ${ }^{2}$ ). But for such applications to emerge, researchers should resist the lure of general rules. We must instead use all the available data to develop tools to study the specific properties of real systems.

The idea that critical transitions should be preceded by warning signals dates as far back as 1984 (ref. 3). In the past six years or so, such signals have been detected in various experiments. For instance, when yeast (Saccharomyces cerevisiae) is grown in chambers, the populations show some recovery after each drop in their nutrient supply as they adapt to the new conditions ${ }^{4}$ But this 'resettling' takes longer as the community approaches a crash. Also, the sizes of populations across chambers can become more variable in space and time ${ }^{5}$ (see 'Predictors of doom').

Such warning signals are tantalizingly general. Researchers have seen the same dynamics - a slower return to equilibrium and increased variance - elsewhere ${ }^{1}$, including in algal overgrowth in lakes, the end of ice ages (tracked by measurements 


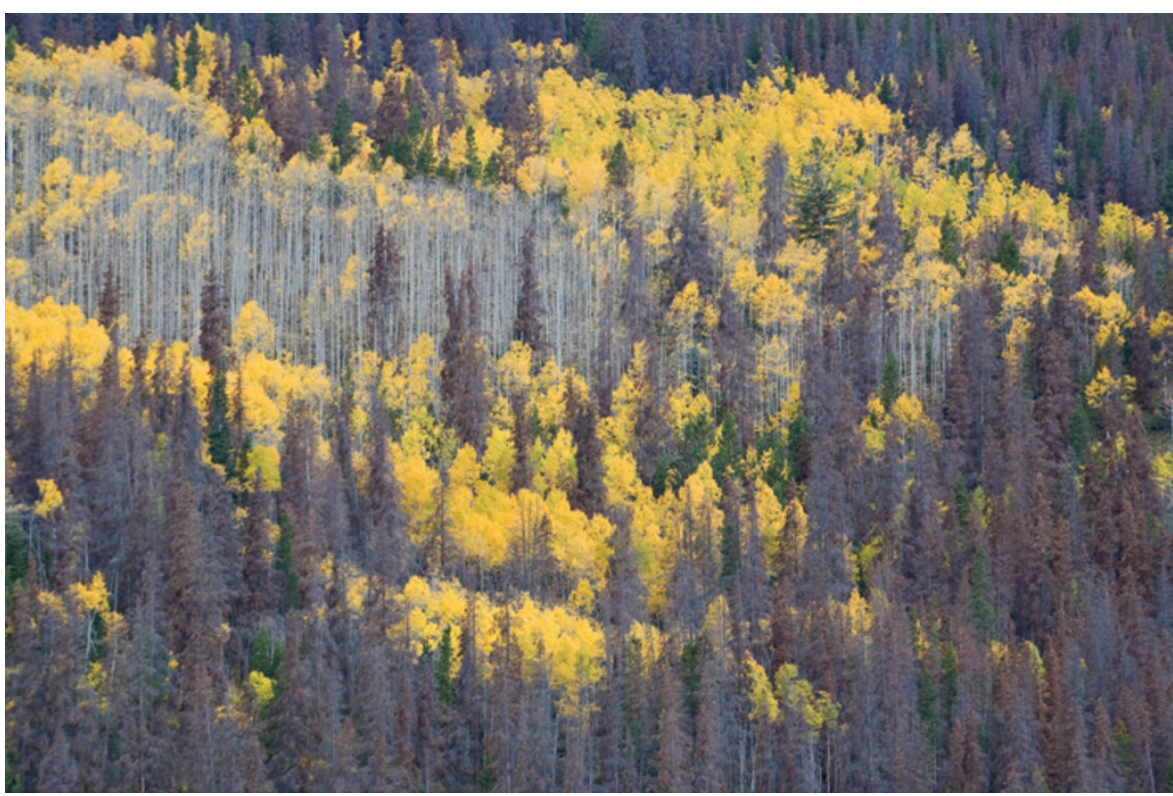

Forests in Colorado are being pushed towards collapse by pine-beetle infestations.

of the dust and atmospheric gas in ice cores) and the onset of epilepsy (signalled by a sudden change in the electrical activity of the brain).

However, no 'one-size-fits-all' property has been found that signals the imminent collapse of a complex system. Hypothetical systems can flip into different states without exhibiting either increased variance or a slower return to equilibrium ${ }^{6,7}$. And other models display exactly the opposite of what is expected. For instance, a model based on the life-history patterns of animals such as salamanders, which spend the juvenile and adult stages of their life cycles in different habitats, suggests that progressive habitat fragmentation can lead to a decrease in the variance of local population sizes ${ }^{7}$.

Much effort is being dedicated to finding 'generic' warning signals that apply across diverse systems. But because the phenomena identified so far are not universally associated with tipping points, nor even sure indicators of a major shift, their predictive power is uncertain. We believe that in most cases, models designed to predict when critical transitions will happen, and in what circumstances, will need to be guided by and perhaps even generated from - data on the specific system of interest.

\section{IMPROVING PREDICTIONS}

Encouragingly, even a small amount of system-specific information can dramatically improve researchers' ability to predict a collapse in simulations. For instance, by incorporating basic knowledge about the birth and death of North Atlantic cod into their models, ecologists were able to double the accuracy with which they predicted an approaching collapse of the population ${ }^{8}$.

Also encouraging is that remote-sensing tools are flooding researchers with gigabytes of data ${ }^{9}$. Satellite images, for instance, provide detailed information about the health (and in some cases, species composition) of vast swathes of forests, including the Amazon rainforest and boreal forests. And electronic data loggers, such as continuousmonitoring floats, are providing detailed information on the physical and biological properties of the world's oceans.

The multidimensional data that these tools provide could lead to modelling approaches with the specificity and statistical power necessary for real-world applications. An as yet untested approach is to plug whatever data are available into machine learning algorithms to find out what, if any, warning signals manifest when certain parameters are adjusted.

Of course, to establish whether a candidate

\section{PREDICTORS OF DOOM}

Before crashing as a result of food-deprivation stress, populations of the water flea Daphnia magna become increasingly variable in size.

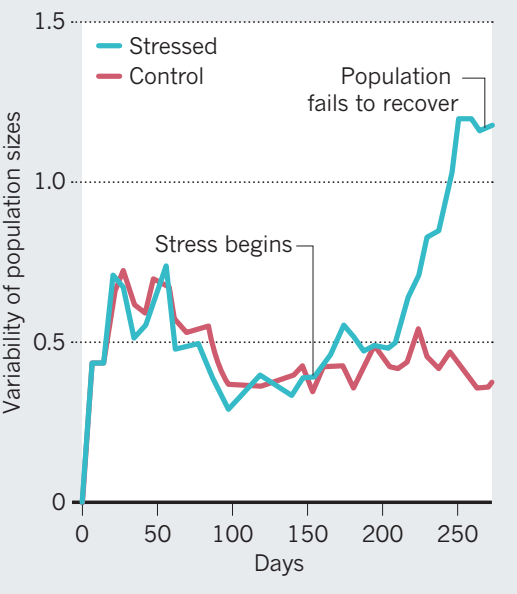

signal is outside the normal range, researchers need to know how the ecosystem, fault line, lake or whatever it is behaves under 'normal' circumstances. Yet controls or replicates are rarely available for natural systems.

One option is to compare the current properties of a system with historical or 'baseline' data. For such baselines to be effective, vast amounts of data must have been collected well before the system nears the tipping point.

Such an approach needs exploring; we don't yet know whether it will even be possible to obtain baseline data for many complex systems. And for ecological systems, comparisons will often be confounded by undocumented changes in data-collection procedures, a switch in management practice or a shift in environmental conditions.

Another way around the lack of controls and replicates, and one that has received less attention, is to model (rather than measure) the expected behaviour of the stable system ${ }^{10}$. In this scenario, various data, including, say, real recordings of the density of invertebrates in a lake, could be fed into a model. In one simulation, a certain parameter, such as the number of predators, would be kept constant; in the other, it would be steadily increased to destabilize the food chain. By comparing various properties of the two systems, modellers could then establish the predictive power of candidate early warning signals.

Both theoretical $^{3}$ and experimental ${ }^{4,5}$ approaches have shown that measurable signs of impending transitions do exist ${ }^{1}$. The challenge now is to resist the seductive but elusive concept of 'generic' warning signals, and focus instead on making predictions about real systems using readily available, or easily collectable, data.

Carl Boettiger is at the Center for Stock Assessment Research, Department of Applied Math and Statistics, University of California, Mail Stop SOE-2, Santa Cruz, California 95064, USA; Alan Hastings is at the Department of Environment Science \& Policy, University of California, Davis, California 95616, USA.

e-mail:cboettig@ucdavis.edu amhastings@ucdavis.edu

1. Scheffer, M. et al. Nature 461, 53-59 (2009).

2. World Economic Forum Global Risks 2013 (in the press).

3. Wissel, C. Oecologia 65, 101-107 (1984).

4. Dai, L., Vorselen, D., Korolev, K. S. \& Gore, J. Science 336, 1175-1177 (2012).

5. Drake, J. M. \& Griffen, B. D. Nature 467, 456-459 (2010).

6. Hastings, A. \& Wysham, D. B. Ecol. Lett. 13, 464-472 (2010)

7. Schreiber, S. \& Rudolf, V. H. W. Ecol. Lett. 11, 576-587 (2008).

8. Lade, S. J. \& Gross, T. PLoS Comput. Biol. 8, e1002360 (2012).

9. Dakos, V., Kéfi, S., Rietkerk, M., van Nes, E. H. \& Scheffer, M. Am. Nat. 177, E153-E166 (2011)

10.Boettiger, C. \& Hastings, A. J. R. Soc. Interface 9 2527-2539 (2012) 